\title{
The Ito Formula for the Ito Processes Driven by the Wiener Processes in a Banach Space
}

\author{
Badri Mamporia \\ Niko Muskhelishvili Institute of Computational Mathematics, Technical University of Georgia, Tbilisi, Georgia
}

Email address:

badrimamporia@yahoo.com (B. Mamporia)

\section{To cite this article:}

Badri Mamporia. The Ito Formula for the Ito Processes Driven by the Wiener Processes in a Banach Space. Pure and Applied Mathematics Journal. Vol. 4, No. 4, 2015, pp. 164-171. doi: 10.11648/j.pamj.20150404.15

\begin{abstract}
Using traditional methods it is possible to prove the Ito formula in a Hilbert space and some Banach spaces with special geometrical properties. The class of such Banach spaces is very narrow-they are subclass of reflexive Banach spaces. Using the definition of a generalized stochastic integral, early we proved the Ito formula in an arbitrary Banach space for the case, when as initial Ito process was the Wiener process. For an arbitrary Banach space and an arbitrary Ito process it is impossible to find the sequence of corresponding step functions with the desired convergence. We consider the space of generalized random processes, introduce general Ito process there and prove in it the Ito formula. Afterward, from the main Ito process in a Banach space we receive the generalized Ito process in the space of generalized random processes and we get the Ito formula in this space. Then we check decompasibilility of the members of the received equality and as they turn out Banach space valued, we get the Ito formula in an arbitrary Banach space. We implemented this approach when the stochastic integral in the Ito process was taken from a Banach space valued non-anticipating random process by the one dimensional Wiener process. In this paper we consider the case, when the stochastic integral is taken from an operator- valued non-anticipating random process by the Wiener process with values in a Banach space.
\end{abstract}

Keywords: Wiener Process in a Banach Space, Covariance Operators, Ito Stochastic Integrals and Ito Processes, the Ito Formula, Stochastic Differential Equations in a Banach Space

\section{Introduction and Preliminaries}

As in the finite dimensional case, the Ito formula plays an important role in the infinite dimensional stochastic analysis. For the cases when the Banach spaces have special geometrical properties, the Ito formula was proved in [1] and [2]. For the Wiener process in an arbitrary separable Banach space, the Ito formula was proved in [3]. The Ito formula for the case, when the stochastic integral that appears in the Ito process is taken from Banach space valued non-anticipating process by the one dimensional Wiener process was proved in [4]. In this paper we prove the Ito formula for the Ito processes when the stochastic integral is taken from the operator valued non-anticipating random processes by the Wiener processes in a Banach space. The main unsolved problem to prove this formula in an arbitrary Banach space is to find such a sequence of step functions converging to the integrand function that their stochastic integrals converge to the stochastic integral from the integrand function. We use the concept of the generalized random element; we consider the space of generalized random processes and introduce the generalized Ito process there. Firstly we prove the Ito formula for the generalized random processes. Then from the initial Ito process in a Banach space we receive a generalized Ito process and write the Ito formula there. Afterward, In the obtained equality we check decomposability of the members of the equality; we found that all of them are Banach space valued. Therefore, we get the Ito formula for the Banach space valued random process. Now we give, some definitions and preliminary results.

Let $X$ be a real separable Banach space, $X^{*}$ - its conjugate, $\mathrm{B}(X)$ - the Borel $\sigma$-algebra of $X,(\Omega, \mathrm{B}, \mathrm{P})$ - a probability space. The continuous linear operator $T: X^{*} \rightarrow \mathrm{L}$ ${ }_{2}(\Omega, B, \mathrm{P})$ is called a generalized random element (GRE) (sometimes it is used the terms: random linear function or cylindrical random element). Denote by $M_{1}:=\mathrm{L}\left(X^{*}, \mathrm{~L}_{2}(\Omega\right.$, $\mathrm{B}, \mathrm{P})$ ) the Banach space of GRE with the norm $\|T\|_{M_{1}}:=\sup _{\| x^{*} \mid \leq 1}\left\|T x^{*}\right\|_{L_{2}}$. A random element (measurable map) 
$\xi: \Omega \rightarrow X$ is said to have a weak second order, if, for all $x^{*} \in X^{*}, \mathrm{E}\left\langle\xi, x^{*}\right\rangle^{2}<\infty$. We can realize the random element $\xi$ as an element of $M_{1}: T_{\xi} \mathrm{x}^{*}=\left\langle\xi, x^{*}\right\rangle$, but not conversely: in infinite dimensional Banach space for all $T: X^{*} \rightarrow \mathrm{L}_{2}$ $(\Omega, B, \mathrm{P})$, there does not always exists the random element $\xi: \Omega \rightarrow X$ such that $T \mathrm{x}^{*}=\left\langle\xi, x^{*}\right\rangle$ for all $x^{*} \in X^{*}$. The problem of existence such random element is the well known problem of decompasibility of the GRE. Denote by $M_{2}$ the linear normed space of all random elements of the weak second order with the norm $\|\xi\|=\left\|T_{\xi}\right\|$. Thus, we can assume $\mathrm{M}_{2} \subset M_{1}$.

Let $\left(\mathrm{W}_{t}\right)_{t \in[0,1]}$ be the Wiener process in a Banach space. That is 1) $\mathrm{W}_{0}=0$ almost surely (a.s.); 2. for all $0 \leq t_{0}<t_{1}<\cdots<t_{n} \leq 1$, the random elements in $X$, $W_{t_{i+1}}-W_{t_{i}}, i=1,2, \cdots, n-1$ are independent; 3. for all $t>s$ $W_{t}-W_{s}$ is a Gaussian random element with a mean 0 and the covariance operator $(t-s) R$, where $R: X^{*} \rightarrow X$ is a fixed Gaussian covariance; 4. $\left(W_{t}\right)_{t \in[0,1]}$ has continuous sample pats.

Let $\left(F_{t}\right)_{t \in[0,1]}$-be an increasing family of $\sigma$-algebras such that a) $W_{t}$ is $F_{t}$-measurable for all $t \in[0,1]$; b) $W_{t}-W_{s}$-is independent of the $\sigma$-algebra $F_{s}$ for all $s<t . F_{0}$ contains all P-null sets in B. In this case we say that $\left(W_{t}\right)_{t \in[0,1]}$ is $F_{t}$ adapted.

If $R: X^{*} \rightarrow X$ is a covariance operator of the random element $W_{1}$, and $R x^{*}=\sum_{k=1}^{\infty}\left\langle a_{k}, x^{*}\right\rangle a_{k},\left(a_{k}\right)_{k \in N} \subset X$, is a representation of the operator $R$ (see [5], Lemma 3.1.1), then there exists the sequence of independent, standard, real valued Wiener processes $\left(w_{k}(t)\right)_{k \in N}$ such that $W_{t}=\sum_{k=1}^{\infty} a_{k} w_{k}(t)$ and the convergence is a. s. uniformly for $t$ in $X$ (see [6 ], Th. 1.4, [7], Th.1.4). We may choose $\left(a_{k}\right)_{k \in N} \subset X$ and $\left(w_{k}(t)\right)_{k \in N}$ such that $\left(w_{k}(t)\right)_{k \in N}$ will be $F_{t}$ adapted for all $k \in N$ (see [7], prop. 2.1).

Let $\left(\mathrm{T}_{t}\right)_{t \in[0,1]}$ be a family of GRE. We call it a generalized random process (GRP). If we have a weak second order random process $\left(\xi_{t}\right)_{t \in[0,1]}, \xi_{t}: \Omega \rightarrow X$, it will be realized as a GRP: $\mathrm{T}_{\xi_{t}} x^{*}=\left\langle\xi_{t}, x^{*}\right\rangle$.

Denote by $G_{R}\left(X^{*}\right)$ the linear space of random functions $\phi: \Omega \rightarrow X^{*}$ such that for all $x \in X\langle\phi(\omega), x\rangle$ is measurable and $\tau_{R}^{2}(\phi) \equiv \int_{\Omega}\langle R \phi(\omega), \phi(\omega)\rangle d \mathrm{P}<\infty . \tau_{R}(\phi)$ is a pseudonorm in $G_{R}\left(X^{*}\right)$.

Definition 1. A function $\phi:[0,1] \times \Omega \rightarrow X^{*}$ is called nonanticipating with respect to $\left(F_{t}\right)_{t \in[0,1]}$ if the function $(t, \omega) \rightarrow\langle\phi(t, \omega), x\rangle \quad$ from $\quad([0,1] \quad \times \Omega, \mathrm{B}[0,1] \times \mathrm{B}) \quad$ into $\left(R^{1}, \mathrm{~B}\left(R^{1}\right)\right)$ is measurable for all $x \in X$, and the function $\omega \rightarrow\langle\phi(t, \omega), x\rangle$ is $F_{t}$-measurable for all $t \in[0,1]$.

By $T G_{R}\left(X^{*}\right)$ we denote the class of non-anticipating random functions $\phi:[0,1] \times \Omega \rightarrow X^{*} \quad$ for which $P_{R}^{2}(\phi) \equiv\left(\int_{0}^{1} \int_{\Omega}\langle R \phi(t, \omega), \phi(t, \omega)\rangle d t d \mathrm{P}<\infty \quad . \quad T G_{R}\left(X^{*}\right) \quad\right.$ is a linear space and $P_{R}$ is a pseudonorm in it.

If $\phi \in T G_{R}\left(X^{*}\right) \quad$ is a step-function $\phi(t, \omega)=\sum_{i=0}^{n-1} \phi_{t_{i}}(\omega) \chi_{\left[t_{i}, t_{i+1}\right)}(t) \quad, \quad 0=t_{0}<t_{1}<\cdots<t_{n}=1$ ， $i=0, \cdots, n-1$, then the stochastic integral of $\phi$ with respect to $\left(W_{t}\right)_{t \in[0,1]}$, is naturally defined by the equality

$$
\int_{0}^{1} \phi(t, \omega) d W_{t}=\sum_{i=0}^{n-1}\left\langle\phi_{t_{i}}(\omega),\left(W_{t_{i}+1}-W_{t_{i}}\right)\right\rangle
$$

The following lemma is true:

Lemma 1 ([7]). For an arbitrary $\phi \in T G_{R}\left(X^{*}\right)$ there exists a sequence of step-functions $\left(\phi_{n}\right)_{n \in N} \subset T G_{R}\left(X^{*}\right)$ such that $\phi_{n} \stackrel{P_{R}}{\rightarrow} \phi$ and $\int_{0}^{1} \phi_{n} d W_{t}$ converges in $L_{2}(\Omega, \mathrm{B}, \mathrm{P})$.

Definition 2 ([7]). Let $\phi \in T G_{R}\left(X^{*}\right)$ and $\left(\phi_{n}\right)_{n \in N} \subset T G_{R}\left(X^{*}\right)$ be step-functions such that $\phi_{n} \stackrel{P_{R}}{\longrightarrow} \phi$ and $\int_{0}^{1} \phi_{n} d W_{t}$ converges in $L_{2}(\Omega, \mathrm{B}, \mathrm{P})$. The limit of the sequence $\int_{0}^{1} \phi_{n} d W_{t}$ is called the stochastic integral of a random function $\phi \in T G_{R}\left(X^{*}\right)$ with respect to the Wiener process $\left(W_{t}\right)_{t \in[0,1]}$ and is denoted by $\int_{0}^{1} \phi d W_{t}$.

Proposition 1. Let $W_{t}=\sum_{k=1}^{\infty} a_{k} w_{k}(t)$ be such a representation of $\left(W_{t}\right)_{t \in[0,1]}$ that $\left(w_{k}(t)\right)_{t \in[0,1]}$ is $F_{t}$-adapted for all $k \in N$, then $\int_{0}^{t} \phi d W_{t}=\sum_{k=1}^{\infty} \int_{0}^{t}\left\langle\phi, a_{k}\right\rangle d w_{k}(t)$ a. s. for all $t \in[0,1]$

Proof. As $\left(w_{k}(t)\right)_{t \in[0,1]}$ is $F_{t}$-adapted for all $k \in N$, the real valued stochastic integrals $\int_{0}^{t}\left\langle\phi, a_{k}\right\rangle d w_{k}(t)$ exist. The sum $\sum_{k=1}^{\infty} \int_{0}^{t}\left\langle\phi, a_{k}\right\rangle d w_{k}(t) \quad$ converges in $L_{2}(\Omega, \mathrm{B}, \mathrm{P}) \quad$ and $E\left(\sum_{k=1}^{\infty} \int_{0}^{t}\left\langle\phi, a_{k}\right\rangle d w_{k}(t)\right)^{2}=\sum_{k=1}^{\infty}\left\langle\phi, a_{k}\right\rangle^{2}=\langle R \phi, \phi\rangle \quad$. Let $\left(\phi_{n}\right)_{n \in N} \subset T G_{R}\left(X^{*}\right)$ be step-functions such that $\phi_{n} \stackrel{P_{R}}{\rightarrow} \phi$ and 
$\int_{0}^{1} \phi_{n} d W_{t}$ converges in $L_{2}(\Omega, \mathrm{B}, \mathrm{P})$. It is easy to see, that the above equality holds for step-functions $\phi_{n}, n \in N$. That is $\int_{0}^{t} \phi_{n} d W_{t}=\sum_{k=1}^{\infty} \int_{0}^{t}\left\langle\phi_{n}, a_{k}\right\rangle d w_{k}(t)$ a. $\quad$ s. for all $t \in[0,1]$. $\sum_{k=1}^{m} \int_{0}^{t}\left\langle\phi_{n}, a_{k}\right\rangle d w_{k}(t)$ converges also to $\sum_{k=1}^{m} \int_{0}^{t}\left\langle\phi, a_{k}\right\rangle d w_{k}(t)$ for all fixed $m \in N$. Therefore, $\sum_{k=1}^{m} \int_{0}^{t}\left\langle\phi_{n}, a_{k}\right\rangle d w_{k}(t) \rightarrow$ $\sum_{k=1}^{m} \int_{0}^{t}\left\langle\phi, a_{k}\right\rangle d w_{k}(t) \rightarrow \sum_{k=1}^{\infty} \int_{0}^{t}\left\langle\phi, a_{k}\right\rangle d w_{k}(t)$, when $m \rightarrow \infty$ and $\sum_{k=1}^{m} \int_{0}^{t}\left\langle\phi_{n}, a_{k}\right\rangle d w_{k}(t) \rightarrow \int_{0}^{t} \phi d W_{t}$ when $n \rightarrow \infty$.

Now consider the linear bounded operator $\phi: X^{*} \rightarrow G_{R}\left(X^{*}\right)$, for all fixed $x^{*} \in X^{*}, \phi x^{*}: \Omega \rightarrow X^{*}$. Denote by $M_{1}{ }^{G} \equiv L\left(X^{*}, G_{R}\left(X^{*}\right)\right)$ the space of such operators with the property: $\tau^{2}{ }_{R}(\phi) \equiv \sup _{\left\|x^{*}\right\| \leq 1} E\left\langle R \phi x^{*}, \phi x^{*}\right\rangle<\infty$. $\tau_{R}(\phi)$ is a pseudonorm in $M_{1}^{G}$. Consider now the family of linear bounded operators $\left(T_{t}\right)_{t \in[0,1]}, T_{t}: X^{*} \rightarrow G_{R}\left(X^{*}\right)$, such that for all $x^{*} \in X^{*}$, the random process $T_{t} x^{*}$ is non - anticipating

and $\tau_{R}^{2}\left(\left(T_{t}\right)_{t \in[0,1]}\right) \equiv$ $\sup _{\left\|x^{*}\right\| \leq 1} \int_{0}^{1} \int_{\Omega}\left\langle R T_{t} x^{*}, T_{t} x^{*}\right\rangle d t d \mathrm{P}<\infty$. Denote by $T M_{1}^{G}$ the space of such family of operators.

Afterward, we will consider the family $\left(T_{t}\right)_{t \in[0,1]}$, $T_{t}: X^{*} \rightarrow G_{R}\left(X^{*}\right) \quad$ with the property $\sup _{\left\|x^{*}\right\| \leq 1} \sup _{\|x\| \leq 1}$ $\int_{0}^{1} \int_{\Omega}\left\langle T_{t} x^{\bullet}, x\right\rangle^{2} d t d \mathrm{P}<\infty$.

Proposition 2. If the family of linear bounded operators $\left(T_{t}\right)_{t \in[0,1]}, T_{t}: X^{*} \rightarrow G_{R}\left(X^{*}\right)$ are such that $\sup _{\| x^{*} \mid \leq 1} \sup _{\|x\| \leq 1}$ $\int_{0}^{1} \int_{\Omega}\left\langle T_{t} x^{*}, x\right\rangle^{2} d t d \mathrm{P}<\infty$, then $\sup _{\left\|x^{*}\right\|^{*} \leq 1} \int_{0}^{1} \int_{\Omega}\left\langle R T_{t} x^{*}, T_{t} x^{*}\right\rangle d t d \mathrm{P}<\infty$, that is $\left(T_{t}\right)_{t \in[0,1]} \in T M_{1}^{G}$.

Proof. . As $R: X^{*} \rightarrow X$ is a Gaussian covariance operator, by the Kwapien-Szymanski's theorem (see [5] p. 262, [8]) there exist the sequences $\left(x_{k}\right)_{k \in N} \subset X$ and $\left(x_{k}^{*}\right)_{k \in n} \subset X^{*}$ such that $\left\langle x_{k}, x_{j}{ }^{*}\right\rangle=\delta_{k, j}, R x_{k}{ }^{*}=x_{k}, k=1,2, \cdots$, for all $x^{*} \in X^{*}, R x^{*}=\sum_{k=1}^{\infty}\left\langle x_{k}, x^{*}\right\rangle x_{k}$ and $\sum_{k=1}^{\infty}\left\|x_{k}\right\|^{2}<\infty$. Then

$$
\begin{aligned}
\sup _{\| x^{*} \mid \leq 1} \int_{0}^{1} \int\left\langle R T_{\Omega} x^{*}, T_{t} x^{*}\right\rangle d t d \mathrm{P}= & \sup _{\| x^{*} \mid \leq 1} \iint_{0}^{1} \int_{\Omega} \sum_{k=1}^{\infty}\left\langle x_{k}, T_{t} x^{*}\right\rangle^{2} d t d \mathrm{P}=\sum_{k=1}^{\infty}\left\|x_{k}\right\| \sup _{\left\|x^{*}\right\| \leq 1} \int_{0}^{1} \int_{\Omega}\left\langle x_{k} /\left\|x_{k}\right\| \|_{t} x^{*}\right\rangle^{2} d t d \mathrm{P} \leq \\
& \sum_{k=1}^{\infty}\left\|x_{k}\right\|^{2} \sup _{\| x \mid \leq 1} \sup _{\left\|x^{*}\right\| \leq 1} \int_{0}^{1} \int_{\Omega}\left\langle T_{t} x^{*}, x\right\rangle^{2} d t d \mathrm{P}<\infty .
\end{aligned}
$$

We can naturally define the stochastic integral from $\left(T_{t}\right)_{t \in[0,1]} \in T M_{1}{ }^{G}$ which is the GRE, defined by the equality $I\left(T_{t}\right)_{t \in[0,1]} x^{*}=\int_{0}^{1} T_{t} x^{*} d W_{t}$. Accordingly, we have the isometrical operator $I: \quad T M_{1}^{G} \rightarrow M_{1}$, $I\left(T_{t}\right)_{t \in[0,1]} x^{*}=\int_{0}^{1} T_{t} x^{*} d W_{t}$.

Lemma 2. For an arbitrary separable-valued $\phi:[0,1] \rightarrow M_{1}^{G}$ there exists a sequence of step-functions $\left(\phi_{n}\right)_{n \in N} \subset T M_{1}^{G}$ such that $\phi_{n} \stackrel{\tau_{R}}{\rightarrow} \phi$ in $T M_{1}^{G}$ and $\int_{0}^{1} \phi_{n} d W_{t}$

$$
\begin{gathered}
\sup _{\left\|x^{*}\right\| \leq 1} \int_{0}^{1} \int_{\Omega} \sum_{k=1}^{\infty}\left\langle\left(\phi x^{*}-\sum_{j=1}^{n}\left\langle\phi x^{*}, x_{j}\right\rangle x_{j}^{*}\right), x_{k}\right\rangle^{2} d t d \mathrm{P}=\sup _{\left\|x^{*}\right\| \leq 1} \int_{0}^{1} \int_{\Omega^{2}} \sum_{k=n+1}^{\infty}\left\langle\phi x^{*}, x_{k}\right\rangle^{2} d t d \mathrm{P} \leq \sum_{k=n+1}^{\infty}\left\|x_{k}\right\|^{2} \sup _{\left\|x^{*}\right\| \leq 1} \iint_{0}^{1}\left\langle\phi x_{\Omega}^{*}, \frac{x_{k}}{\left\|x_{k}\right\|}\right\rangle^{2} d t d \mathrm{P} \leq \\
\sum_{k=n+1}^{\infty}\left\|x_{k}\right\|^{2} \sup _{\left\|x^{*}\right\| \leq 1} \sup _{\|x\| \leq 1} \int_{0}^{1} \int_{\Omega}\left\langle\phi x^{*}, x\right\rangle^{2} d t d \mathrm{P} \rightarrow 0 .
\end{gathered}
$$

Proof. Analogous to the case of proposition 2, consider the sequences $\left(x_{k}\right)_{k \in N} \subset X$ and $\left(x_{k}^{*}\right)_{k \in n} \subset X^{*}$ such that $\left\langle x_{k}, x_{j}{ }^{*}\right\rangle=\delta_{k, j}, R x_{k}{ }^{*}=x_{k}, k=1,2, \cdots$, for all $x^{*} \in X^{*}$, $R x^{*}=\sum_{k=1}^{\infty}\left\langle x_{k}, x^{*}\right\rangle x_{k}$ and $\sum_{k=1}^{\infty}\left\|x_{k}\right\|^{2}<\infty$. Let $\phi \in T M_{1}{ }^{G}$ and denote by $f_{n}(t) x^{*}=\sum_{k=1}^{n}\left\langle\phi(t) x^{*}, x_{k}\right\rangle x_{k}{ }^{*}, n=1,2, \cdots$. the functions from $T M_{1}^{G}$.
For any fixed $k$, consider now the GRP $\left(\varphi_{k}(t)\right)_{t \in[0,1]} \equiv\left\langle\phi(t) x^{*}, x_{k}\right\rangle, \varphi_{k}(t): X^{\bullet} \rightarrow L_{2}(\Omega, \mathrm{B}, \mathrm{P})$, so we have the map $\varphi_{k}:[0,1] \rightarrow M_{1}, \int_{0}^{1}\left\|\varphi_{k}\right\|_{M_{1}}{ }^{2} d t<\infty$ and $\varphi_{k}$ is separable-valued, therefore, by the lemma 1 from [9], there exists the sequence of non-anticipating step functions $\varphi_{k m}:[0,1] \rightarrow M_{1}$ such that $\int_{0}^{1}\left\|\varphi_{k}(t)-\varphi_{k m}(t)\right\|_{M_{1}}^{2} d t \rightarrow 0$. 
Afterward for $f_{n}(t) x^{*}=\sum_{k=1}^{n}\left\langle\phi(t) x^{*}, x_{k}\right\rangle x_{k}{ }^{*}$, the sequence $f_{n m} \equiv \sum_{k=1}^{n} \varphi_{k m}(t) x_{k}^{\cdot}$ is such that $\tau_{R}^{2}\left(f_{n m}-f_{n}\right) \rightarrow 0$, when $m \rightarrow \infty$. Therefore, we may choose a sequence of stepfunctions $\left(\phi_{n}\right)_{n \in N} \subset T M_{1}^{G}$ such that $\phi_{n} \stackrel{\tau_{R}}{\rightarrow} \phi$. It is easy to see, that $\int_{0}^{1} \phi_{n} d W_{t}$ converges in $M_{1}$ to the $\int_{0}^{1} \phi d W_{t}$.

If we have $F(t, \omega):[0,1] \times \Omega \rightarrow L\left(M_{1}, M_{1}\right)$ nonanticipating operator valued random process and nonanticipating $\left(T_{t}\right)_{t \in[0,1]} \in T M_{1}^{G}$, then we can define the generalized stochastic integral $I: X^{*} \rightarrow L_{2}(\Omega, \mathrm{B}, \mathrm{P})$,

$$
I x^{*}=\int_{0}^{1} F(t, \omega) T_{t} x^{*} d W_{t} \equiv \sum_{m=1}^{\infty} \int_{0}^{1} F(t, \omega)\left\langle T_{t} x^{*}, x_{k}\right\rangle d w_{m}(t)
$$

as $\left\langle T_{t}(\cdot), x\right\rangle: X^{*} \rightarrow L_{2}(\Omega, \mathrm{B}, \mathrm{P})$ is a generalized random process. Here we use the representation of the Wiener process in a Banach space by the sum of one dimensional, independent, non-anticipating Wiener processes, and $\left(x_{k}\right)_{k \in N}$ is such that $R x^{*}=\sum_{k=1}^{\infty}\left\langle x^{*}, x_{k}\right\rangle x_{k}$ is the representation of the covariance operator of $W_{1}$. Let now $X$ be a separable Banach space and $L(X, X)$ be the space of bounded linear operators from $X$ to $X$. We will consider the random processes $\xi_{t}: \Omega \rightarrow L(X, X)$ such that $\int_{0}^{1} \int_{\Omega}\left\langle\xi_{t}(\omega) x, x^{\bullet}\right\rangle^{2} d t d \mathrm{P}<\infty$ for all $x \in X \quad$ and $x^{\bullet} \in X^{\bullet}$.

Proposition 2. If the random process $\xi_{t}: \Omega \rightarrow L(X, X)$ is such, that $\int_{0}^{1} \int_{\Omega}\left\langle\xi_{t}(\omega) x, x^{\bullet}\right\rangle^{2} d t d \mathrm{P}<\infty$ for all $x \in X \quad$ and $x^{\bullet} \in X^{\bullet}$, then $\sup _{\left\|x^{*}\right\| \leq 1} \int_{0}^{1} \int_{\Omega}\left\langle R \xi_{t}^{\bullet}(\omega) x^{\bullet}, \xi_{t}(\omega) x^{\bullet}\right\rangle^{2} d t d \mathrm{P}<\infty$, where the operator $\xi_{t}{ }_{t}(\omega)$ is conjugate of the operator $\xi_{t}(\omega)$.

Proof. Firstly we prove that $\sup _{\left\|x^{*}\right\| \leq 1} \sup _{\|x\| \leq 1} \int_{0}^{1} \int_{\Omega}\left\langle\xi_{t}(\omega) x, x^{*}\right\rangle^{2} d t d \mathrm{P}<\infty$. Consider the family of linear operators $T_{x}: X^{\bullet} \rightarrow L_{2}([0,1] \times \Omega, \mathrm{B}[0,1] \times \mathrm{B}, \lambda \times \mathrm{P})$, $T_{x} x^{\bullet}=\left\langle\xi_{t}(\omega) x, x^{\bullet}\right\rangle, x \in B(X) \equiv\{x:\|x\| \leq 1\}$. From the closed graph theorem it follows that $T_{x}$ is a continuous operator for all fixed $x \in X$. That is $\left\{T_{x}, x \in B(X)\right\}$ is a collection of continuous linear operators from $X^{\bullet}$ to $L_{2}([0,1], \Omega)$. For all fix $x^{\bullet} \in X^{\bullet}$, if we consider the linear operator $S: X \rightarrow L_{2}([0,1], \Omega), S x=\left\langle\xi_{t}(\omega) x, x^{*}\right\rangle$, by the closed graph theorem, we can proof boundedness of the operator $S$. That is, for all fixed $x^{\bullet} \in X^{\bullet}, \sup _{x \in B(X)}\left\|T_{x}\left(x^{\bullet}\right)\right\|^{2}=\sup _{\|x\| \leq 1}\|S(x)\|^{2}$ $=\sup _{\|x\| \leq 1} \int_{0}^{1} \int_{\Omega}\left\langle\xi_{t}(\omega) x, x^{\bullet}\right\rangle^{2} d t d \mathrm{P}<\infty$. Then, by the uniform boundedness $\sup _{\left\|x^{*}\right\| \leq 1} \sup _{\|x\| \leq 1} \int_{0}^{1} \int_{\Omega}\left\langle\xi_{t}(\omega) x, x^{*}\right\rangle^{2} d t d \mathrm{P}<\infty$. principle,

Let now the sequences $\left(x_{k}\right)_{k \in N} \subset X$ and $\left(x_{k}{ }^{*}\right)_{k \in n} \subset X^{*}$ be such that $\left\langle x_{k}, x_{j}^{*}\right\rangle=\delta_{k, j}, R x_{k}{ }^{*}=x_{k}, k=1,2, \cdots$, for all $x^{*} \in X^{*}, R x^{*}=\sum_{k=1}^{\infty}\left\langle x_{k}, x^{*}\right\rangle x_{k}$ and $\sum_{k=1}^{\infty}\left\|x_{k}\right\|^{2}<\infty$. Then we have

$$
\begin{aligned}
& \sup _{\left\|x^{*}\right\| \leq 1} \int_{0}^{1} \int_{\Omega}\left\langle R \xi_{t}(\omega) x^{\bullet}, \xi^{\bullet} x^{\bullet}\right\rangle^{2} d t d \mathrm{P}=\sup _{\|\|^{*} \| \leq 1} \int_{0}^{1} \int_{\Omega} \sum_{k=1}^{\infty}\left\langle\xi_{t}(\omega) x^{\bullet}, x_{k}\right\rangle^{2} d t d \mathrm{P}= \\
& \sum_{k=1}^{\infty}\left\|x_{k}\right\|^{2} \sup _{\left\|x^{*}\right\| \leq 1} \int_{0}^{1} \int_{\Omega}\left\langle\left\langle\xi_{t} \cdot(\omega) x^{\bullet}, \frac{x_{k}}{\left\|x_{k}\right\|}\right\rangle^{2} d t d \mathrm{P} \leq \sum_{k=1}^{\infty}\left\|x_{k}\right\|^{2} \sup _{\| x^{*} \mid \leq 1} \sup _{\|x\| \leq 1} \int_{0}^{1} \int_{\Omega}\left\langle\xi_{t}(\omega) x, x^{*}\right\rangle^{2} d t d \mathrm{P}<\infty\right.
\end{aligned}
$$

Definition 3. The random process $\xi_{t}: \Omega \rightarrow L(X, X)$ is non-anticipating with respect to the family of the $\sigma$-algebra $\left(F_{t}\right)_{t \in[0,1]}$ if, for all $x \in X, \quad \xi_{t}(\omega) x:[0,1] \times \Omega \rightarrow X$ is measurable and, for all $t \in[0,1]$, the random element $\xi_{t} x: \Omega \rightarrow X$ is $F_{t}$-measurable.

Definition 4. We say that the non-anticipating random process $\left(\xi_{t}\right)_{t \in[0,1]}, \xi_{t}: \Omega \rightarrow L(X, X)$ belongs to the class $T G_{R}(L(X, X))$ if

$$
\tau^{2}(\xi) \equiv \sup _{\left\|x^{*}\right\| \leq 1} \int_{0}^{1} \int_{\omega}\left\langle R \xi_{t}^{*}(\omega) x^{*}, \xi_{t}^{*}(\omega) x^{*}\right\rangle d t d \mathrm{P}<\infty,
$$

where $\xi_{t}^{*}(\omega)$ is the linear operator, conjugate to the operator $\xi_{t}(\omega) . T G_{R}(L(X, X))$ is a linear space with the pseudonorm $\tau(\xi)$.

Let $\xi \in T G_{R}(L(X, X))$ and $x^{*} \in X^{*}$. Then $\xi^{*} x^{*}:[0,1] \times \Omega \rightarrow X^{*} \quad$ is non-anticipating and $\int_{0}^{1} \int_{\omega}\left\langle R \xi_{t}^{*}(\omega) x^{*}, \xi_{t}^{*}(\omega) x^{*}\right\rangle d t d \mathrm{P}<\infty$. We can define the stochastic integral $\int_{0}^{1} \xi_{t}^{*}(\omega) x^{*} d W_{t}$, which is the random variable with a mean 0 and variance $\int_{0}^{1} \int_{\omega}\left\langle R \xi_{t}^{*}(\omega) x^{*}, \xi_{t}^{*}(\omega) x^{*}\right\rangle d t d \mathrm{P}$. Therefore, we can consider 
the GRE $I_{\xi}: X^{*} \rightarrow L_{2}(\Omega, \mathrm{B}, \mathrm{P}), I_{\xi} x^{*}=\int_{0}^{1} \xi_{t}^{*}(\omega) x^{*} d W_{t}$.

Definition 5. The generalized random element $I_{\xi} x^{*}=$ $\int_{0}^{1} \xi_{t}^{*}(\omega) x^{*} d W_{t}$ is called the generalized stochastic integral from the random process $\xi \in T G_{R}(L(X, X))$. If there exists the random element $\eta: \Omega \rightarrow X$ such that $\left\langle\eta, x^{*}\right\rangle=I_{\xi} x^{*}=$ $\int_{0}^{1} \xi_{t}^{*}(\omega) x^{*} d W_{t}$ for all $x^{*} \in X^{*}$, then we say that there exists the stochastic integral from the operator-valued nonanticipating random process $\left(\xi_{t}\right)_{t \in[0,1]}, \xi_{t}: \Omega \rightarrow L(X, X)$ by the Wiener process in a Banach space $X$ and then we write $\eta=\int_{0}^{1} \xi_{t}(\omega) d W_{t}$.

\section{The Ito Formula}

We will prove the Ito formula for the generalized Ito processes and, as a consequence, we receive the Ito formula for the Banach space valued Ito processes, where the stochastic integral is taken from the operator-valued random process by the Wiener processes in a Banach space.

Definition 4. A non-anticipating GRP is called the Generalized Ito process, if there exist non-anticipating GRP $(a(t))_{t \in[0,1]}, a(t): X^{*} \rightarrow L_{2}(\Omega, \mathrm{B}, \mathrm{P}), \int_{0}^{1}\|a(t)\|_{M_{1}}{ }^{2} d t<\infty$, and non-anticipating $\quad(B(t))_{t \in[0,1]} \quad \in T M_{1}{ }^{G}$ $\sup _{\left\|x^{*}\right\| \leq 1} \int_{0}^{1} \int_{\Omega}\left\langle R B(t) x^{*}, B(t) x^{*}\right\rangle d t d \mathrm{P}<\infty \quad$, such that, for all $x^{*} \in X^{*}$,

$$
\mathrm{T}_{t} x^{*}=T_{0} x^{*}+\int_{0}^{t} a(s) x^{*} d s+\int_{0}^{t} B(s) x^{*} d W_{s} \text { a.s. }
$$

Lemma 3. Let the generalized Ito process $T_{t} x^{*}=T_{0} x^{*}+\int_{0}^{t} a(s) x^{*} d s+\int_{0}^{t} B(s) x^{*} d W_{s} \quad$ be such that $a:[0,1] \rightarrow M_{1}$ and $B:[0,1] \rightarrow M_{1}{ }^{G}$ are separable-valued, then there exist the sequences of non-anticipating step functions $\quad\left(a_{n}\right)_{n \in N}$ and $\left(B_{n}\right)_{n \in N}$ such that $\int_{0}^{1}\left\|a_{n}(t)-a(t)\right\|_{M_{1}}{ }^{2} d t \rightarrow 0 \quad, \quad \int_{0}^{1}\left\|B_{n}(t)-B(t)\right\|_{M_{1}{ }{ }^{2}} d t \rightarrow 0 \quad$ and $\left\|T^{(n)}{ }_{t}-T_{t}\right\|_{M_{1}}{ }^{2} \rightarrow 0$ uniformly for $t$, where

$$
\mathrm{T}^{(n)}{ }_{t} x^{*}=T_{0} x^{*}+\int_{0}^{t} a_{n}(s) x^{*} d s+\int_{0}^{t} B_{n}(s) x^{*} d W_{s} .
$$

Proof. The existence of the sequences of non-anticipating step functions $\left(a_{n}\right)_{n \in N}$ is proved in [ 3 ] ( lemma 1) and existence of the sequence of non-anticipating step functions
$\left(B_{n}\right)_{n \in N}$ follows from the lemma 2. Uniformness of the convergence for $t$ of $\left\|T^{(n)}{ }_{t}-T_{t}\right\|_{M_{1}}{ }^{2} \rightarrow 0$ follows from the inequality

$$
\left\|T^{(n)}{ }_{t}-T_{t}\right\|_{M_{1}}{ }^{2} \leq\left\|T_{1}^{(n)}-T_{1}\right\|_{M_{1}}{ }^{2} \rightarrow 0
$$

Theorem 1(Formula Ito). Let $\mathrm{T}$ ${ }_{t} x^{*}=T_{0} x^{*}+\int_{0}^{t} a(s) x^{*} d s+\int_{0}^{t} B(s) x^{*} d W_{s}$ be a generalized Ito process, where $a$ and $B$ are separable-valued nonanticipating GRP such that $\int_{0}^{1}\|a(t)\|_{M_{1}}{ }^{2} d t<\infty$, $\int_{0}^{1}\|B(t)\|_{M_{1}{ }^{G}}{ }^{2} d t<\infty$. Let $f:[0,1] \times M_{1} \rightarrow M_{1}$ be a continuous function such that the derivatives $f_{t}^{\prime}:[0,1] \times M_{1} \rightarrow M_{1}$, $f_{T}^{\prime}:[0,1] \times M_{1} \rightarrow L\left(M_{1}, M_{1}\right) \quad$ and $\quad f_{T, T}^{\prime \prime}:[0,1] \times M_{1} \rightarrow L$ $\left(M_{1}, L\left(M_{1}, M_{1}\right)\right.$ are continuous. Then

$$
\begin{gathered}
f\left(t, T_{t}\right)=f\left(0, T_{0}\right)+\int_{0}^{t} f_{t}^{\prime}\left(s, T_{s}\right) d s+\int_{0}^{t} f_{T}^{\prime}\left(s, T_{s}\right) a(s) d s+ \\
\frac{1}{2} \int_{0}^{t}\left(\sum_{k=1}^{\infty} f^{\prime \prime}{ }_{T, T}\left(s, T_{s}\right) B(s) a_{k} B(s) a_{k}\right) d s+\int_{0}^{t} f_{T}^{\prime}\left(s, T_{s}\right) B(s) d W(s) .
\end{gathered}
$$

Proof. As in the finite dimensional case, we show that it is enough to prove this theorem for the step functions $a$ and $B$ : let $\left(a_{n}\right)_{n \in N}$ and $\left(B_{n}\right)_{n \in N}$ be the sequences of step functions such that $\int_{0}^{1}\left\|a_{n}(t)-a(t)\right\|_{M_{1}}{ }^{2} d t \quad \rightarrow 0 \quad$ and $\int_{0}^{1}\left\|B_{n}(t)-B(t)\right\|_{M_{1}{ }^{G}}{ }^{2} d t \rightarrow 0, \quad$ then $\quad, \quad\left\|T^{(n)}{ }_{t}-T_{t}\right\|_{M_{1}}{ }^{2} \rightarrow 0$ uniformly for $\mathrm{t}$, where

$$
T_{t}^{(n)} x^{*}=T_{0} x^{*}+\int_{0}^{t} a_{n}(s) x^{*} d s+\int_{0}^{t} B_{n}(s) x^{*} d W_{s} .
$$

Let the Ito formula be true for the step functions:

$$
\begin{gathered}
f\left(t, T_{t}^{(n)}\right)=f\left(0, T_{0}\right)+\int_{0}^{t} f_{t}^{\prime}\left(s, T_{s}^{(n)}\right) d s+\int_{0}^{t} f_{T}^{\prime}\left(s, T_{s}^{(n)}\right) a_{n}(s) d s+ \\
\frac{1}{2} \int_{0}^{t}\left(\sum_{k=1}^{\infty} f^{\prime \prime}{ }_{T, T}\left(s, T^{(n)}{ }_{s}\right) B(s) a_{k} B(s) a_{k}\right) d s+ \\
\int_{0}^{t} f_{T}^{\prime}\left(s, T_{s}^{(n)}\right) B(s) d W(s) . .
\end{gathered}
$$

As $f_{t}^{\prime}\left(s, T_{s}^{(n)}\right)$ are continuous functions on $[0,1]$ converging to the continuous function $f_{t}^{\prime}\left(s, T_{s}\right)$, then they are bounded. Thereby, by the Lebesgue theorem, we have the 
convergence $\int_{0}^{t} f_{t}^{\prime}\left(s, T_{s}^{(n)}\right) d s \rightarrow \int_{0}^{t} f_{t}^{\prime}\left(s, T_{s}\right) d s$. Furthermore,

we have

$$
\begin{gathered}
\int_{0}^{t}\left\|f_{T}^{\prime}\left(s, T_{s}^{(n)}\right) a_{n}(s)-f_{T}^{\prime}\left(s, T_{s}\right) a_{s}\right\| d s \leq \int_{0}^{1}\left\|f_{T}^{\prime}\left(s, T_{s}^{(n)}\right)\right\|\left\|a_{n}(s)-a(s)\right\| d s++\int_{0}^{1}\|a(s)\|\left\|f_{T}^{\prime}\left(s, T_{s}^{(n)}\right)-f_{T}^{\prime}\left(s, T_{s}\right)\right\| d s \leq \mathrm{C} \\
\int_{0}^{1}\left\|a_{n}(s)-a(s)\right\| d s++\left(\int_{0}^{1}\|a(s)\|^{2} d s\right)^{1 / 2}\left(\int_{0}^{1}\left\|f_{T}^{\prime}\left(s, T_{s}^{(n)}\right)-f_{T}^{\prime}\left(s, T_{s}\right)\right\|^{2} d s\right)^{1 / 2} \rightarrow 0 ;
\end{gathered}
$$

In principle, we can similarly prove

and

$$
\frac{1}{2} \int_{0}^{t}\left(\sum_{k=1}^{\infty} f^{\prime \prime}{ }_{T, T}\left(s, T_{s}^{(n)}\right) B(s) a_{k} B(s) a_{k}\right) d s \rightarrow \frac{1}{2} \int_{0}^{t}\left(\sum_{k=1}^{\infty} f^{\prime \prime}{ }_{T, T}\left(s, T_{s}\right) B(s) a_{k} B(s) a_{k}\right) d s
$$

$\int_{0}^{t} f_{T}^{\prime}\left(s, T_{s}^{(n)}\right) B(s) d W(s) \quad \rightarrow \quad \int_{0}^{t} f_{T}^{\prime}\left(s, T_{s}\right) B(s) d W(s) \quad$.

Therefore, it is enough to prove the Ito lemma for the step functions and, by additivity of integrals, we need to prove it when $T_{t}=T_{0}+a t+B W_{t}$, where $a$ and $B$ are the elements of $M_{1}$ and $M_{1}{ }^{G}$ correspondingly. For simplicity, we can assume that $T_{0}=0$. Then the function $u\left(t, B W_{t}\right)=f\left(t, a t+B W_{t}\right)$ has the same smoothness as $f$ and so, it is enough to prove the Ito formula for function $u\left(t, B W_{t}\right)$. Let $l=\left[2^{n} t\right], \quad \Delta W=W_{\frac{k}{2^{n}}}-W_{\frac{(k-1)}{2^{n}}}, \Delta=\frac{1}{2^{n}}$, $n=1,2, \ldots$ Then, by the Taylor's formula, we have

$$
\begin{gathered}
u\left(t, B W_{t}\right)-u(0,0)=\sum_{k \leq l} u\left(\frac{k}{2^{n}}, B W_{\frac{k}{2^{n}}}\right)-u\left(\frac{k-1}{2^{n}}, B W_{\frac{k-1}{2^{n}}}\right)+u\left(t, B W_{t}\right)-u\left(\frac{l}{2^{n}}, B W_{\frac{l}{2^{n}}}\right)=\sum_{k \leq l} \int_{0}^{1} u_{t}^{\prime}\left(\frac{k-1}{2^{n}}+\frac{s}{2^{n}}, B W_{\frac{k-1}{2^{n}}}\right) \cdot \frac{1}{2^{n}} d s+ \\
\sum_{k \leq l} u_{T}^{\prime}\left(\frac{k-1}{2^{n}}, B W_{\frac{k-1}{2^{n}}}\right) B \Delta W+\sum_{k \leq l}^{1} \int_{0}^{1}(1-s) u_{T T}^{\prime \prime}\left(\frac{k-1}{2^{n}}, B W_{\frac{k-1}{2^{n}}}+s B \Delta W\right) B \Delta W B \Delta W d s+u\left(t, B W_{t}\right)-u\left(\frac{l}{2^{n}}, B W_{\frac{l}{2^{n}}}\right)= \\
\sum_{k \leq l} u_{t}^{\prime}\left(\frac{k-1}{2^{n}}, B W_{\frac{k-1}{2^{n}}}\right) \cdot \frac{1}{2^{n}}+\sum_{k \leq l}^{1} \int_{0}^{1}\left[u_{t}^{\prime}\left(\frac{k-1}{2^{n}}+\frac{s}{2^{n}}, B W_{\frac{k-1}{2^{n}}}\right)-u_{t}^{\prime}\left(\frac{k-1}{2^{n}}, B W_{\frac{k-1}{2^{n}}}\right)\right] \cdot \frac{1}{2^{n}} d s+\sum_{k \leq l} u_{T}^{\prime}\left(\frac{k-1}{2^{n}}, B W_{\frac{k-1}{2^{n}}}\right) B \Delta W+ \\
\left.\frac{1}{2} \sum_{k \leq l} u_{T T}^{\prime \prime}\left(\frac{k-1}{2^{n}}, B W_{\frac{k-1}{2^{n}}}\right)\right] \cdot B \Delta W B \Delta W+\sum_{k \leq l}^{1} \int_{0}^{1}(1-s)\left[u_{T T}^{\prime \prime}\left(\frac{k-1}{2^{n}}, B W_{\frac{k-1}{2^{n}}}+s B \Delta W\right)-u_{T T}^{\prime \prime}\left(\frac{k-1}{2^{n}}, B W_{\frac{k-1}{2^{n}}}\right)\right] B \Delta W B \Delta W d s+ \\
u\left(t, B W_{t}\right)-u\left(\frac{l}{2^{n}}, B W_{\frac{l}{2^{n}}}\right)=\sum_{k \leq l} u_{t}^{\prime}\left(\frac{k-1}{2^{n}}, B W_{\frac{k-1}{2^{n}}}\right) \cdot \frac{1}{2^{n}}+\sum_{k \leq l} u_{T}^{\prime}\left(\frac{k-1}{2^{n}}, B W_{\frac{k-1}{2^{n}}}\right) B \Delta W+ \\
\frac{1}{2} \sum_{k \leq l} \frac{1}{2^{n}} \sum_{m=1}^{\infty} u_{T T}^{\prime \prime}\left(\frac{k-1}{2^{n}}, B W_{\frac{k-1}{2^{n}}}\right) B x_{m} B x_{m}+A_{n}+B_{n}+C_{n}+\delta_{n},
\end{gathered}
$$

where

$$
\begin{gathered}
A_{n}=\sum_{k \leq l} \int_{0}^{1}\left[u_{t}^{\prime}\left(\frac{k-1}{2^{n}}+\frac{s}{2^{n}}, B W_{\frac{k-1}{2^{n}}}\right)-u_{t}^{\prime}\left(\frac{k-1}{2^{n}}, B W_{\frac{k-1}{2^{n}}}\right) \cdot \frac{1}{2^{n}} d s,\right. \\
B_{n}=\sum_{k \leq l} \int_{0}^{1}(1-s) u_{T T}^{\prime \prime}\left(\frac{k-1}{2^{n}}, B W_{\frac{k-1}{2^{n}}}+s B \Delta W\right) B \Delta W B \Delta W d s-\frac{1}{2} u_{T T}^{\prime \prime}\left(\frac{k-1}{2^{n}}, B W_{\frac{k-1}{2^{n}}}\right) B \Delta W B \Delta W, \\
C_{n}=\frac{1}{2} \sum_{k \leq l} u_{T T}^{\prime \prime}\left(\frac{k-1}{2^{n}}, B W_{\frac{k-1}{2^{n}}}\right) B \Delta W B \Delta W-\frac{1}{2} \sum_{k \leq l} \frac{1}{2^{n}} \sum_{m=1}^{\infty} u_{T T}^{\prime \prime}\left(\frac{k-1}{2^{n}}, B W_{\frac{k-1}{2^{n}}}\right) B x_{m} B x_{m}, \\
\delta_{n}=u\left(t, B W_{t}\right)-u\left(\frac{l}{2^{n}}, B W_{\frac{l}{2^{n}}}\right)
\end{gathered}
$$

and $\left(x_{k}\right)_{k \in N} \subset X$ is such, that there exists $\left(x_{k}^{*}\right)_{k \in n} \subset X^{*},\left\langle x_{k}, x_{j}^{*}\right\rangle=\delta_{k, j}, R x_{k}^{*}=x_{k}, k=1,2, \cdots$, for all $x^{*} \in X^{*}$, $R x^{*}=\sum_{k=1}^{\infty}\left\langle x_{k}, x^{*}\right\rangle x_{k}$ and $\sum_{k=1}^{\infty}\left\|x_{k}\right\|^{2}<\infty$. 
Using the technique developed in [ 3], (theorem 1) it is not difficult to prove, that $\left\|A_{n}\right\|_{M_{1}} \rightarrow 0, B_{n} x^{*} \rightarrow 0, C_{n} x^{*} \rightarrow 0$ and $\delta_{n} x^{*} \rightarrow 0$ for all $x^{*} \in X^{*}$ in $L_{2}(\Omega, \mathrm{B}, \mathrm{P})$. Therefore, we have

$$
u\left(t, B W_{t}\right) x^{*}=u(0,0) x^{*}+\int_{0}^{t} u_{t}^{\prime}\left(s, B W_{s}\right) x^{*} d s+\int_{0}^{t} \sum_{m=1}^{\infty}\left\langle u_{T}^{\prime}\left(s, B W_{s}\right) B x^{*}, x_{m}\right\rangle d w_{m}(s)+\frac{1}{2} \sum_{m=1}^{\infty} \int_{0}^{t}\left\langle u_{T T}^{\prime \prime}\left(s, B W_{s}\right) B x_{m} B x^{*}, x_{m}\right\rangle d s
$$

for all $x^{*} \in X^{*}$. That is, we have the equality in $M_{1}$ :

$$
u\left(t, B W_{t}\right)=u(0,0)+\int_{0}^{t} u_{t}^{\prime}\left(s, B W_{s}\right) d s+\int_{0}^{t} u_{T}^{\prime}\left(s, B_{s}\right) B d W(s)+\frac{1}{2} \sum_{m=1}^{\infty} \int_{0}^{t} u^{\prime \prime}{ }_{T T}\left(s, B W_{s}\right) B x_{m} B x_{m} d s .
$$

Now let us return to the function $f$ and remember that $f\left(t, a t+B W_{t}\right)=u\left(t, B W_{t}\right)$, then $u_{t}^{\prime}\left(t, B W_{t}\right)=f_{t}^{\prime}\left(t, a t+B W_{t}\right)+f_{T}^{\prime}\left(t, a t+B W_{t}\right) a$. Therefore, we have

$$
f\left(t, T_{t}\right)=f\left(0, T_{0}\right)+\int_{0}^{t} f_{t}^{\prime}\left(s, T_{s}\right) d s+\int_{0}^{t} f_{T}^{\prime}\left(s, T_{s}\right) a(s) d s+\frac{1}{2} \sum_{m=1}^{\infty} \int_{0}^{t} f_{T T}^{\prime \prime}\left(s, T_{s}\right) B(s) x_{m} B(s) x_{m} d s++\int_{0}^{t} f_{T}^{\prime}\left(s, T_{s}\right) B(s) d W_{s},
$$

where $\left(x_{k}\right)_{k \in N} \subset X$ is such, that there exists $\left(x_{k}^{*}\right)_{k \in n} \subset X^{*}$, $\left\langle x_{k}, x_{j}^{*}\right\rangle=\delta_{k, j}, R x_{k}^{*}=x_{k}, k=1,2, \cdots$, for all

$$
x^{*} \in X^{*}, R x^{*}=\sum_{k=1}^{\infty}\left\langle x_{k}, x^{*}\right\rangle x_{k} \text { and } \sum_{k=1}^{\infty}\left\|x_{k}\right\|^{2}<\infty .
$$

Let the generalized Ito process $\mathrm{T}$ ${ }_{t} x^{*}=T_{0} x^{*}+\int_{0}^{t} a(s) x^{*} d s+\int_{0}^{t} B(s) x^{*} d W_{s}$ be such that there exists the $\mathrm{X}$-valued random process $\left(\xi_{t}\right)_{t \in[0,1]}$ with property $\left\langle\xi_{t}, x^{*}\right\rangle=T_{t} x^{*} \quad$ for $\quad$ all $\quad x^{*} \in X^{*} \quad$ and

$$
\begin{aligned}
\left\langle f\left(t, \xi_{t}\right), x^{*}\right\rangle=\left\langle f\left(0, \xi_{0}\right), x^{*}\right\rangle+\left\langle\int_{0}^{t} f_{t}^{\prime}\left(s, \xi_{s}\right) d s, x^{*}\right\rangle & +\left\langle\int_{0}^{t} f_{T}^{\prime}\left(s, \xi_{s}\right) a(s) d s, x^{*}\right\rangle+\left\langle\frac{1}{2} \int_{0}^{t} \sum_{m=1}^{\infty}\left\langle f_{T T}^{\prime \prime}\left(s, \xi_{s}\right) B(s) x_{m} B(s) x^{*}, x_{m}\right\rangle d s,\right\rangle+ \\
& \int_{0}^{t}\left\langle\left(f_{T}^{\prime}\left(s, \xi_{s}\right) B(s)\right)^{*} x^{*}\right\rangle d W_{s} .
\end{aligned}
$$

$\xi_{t}=\xi_{0}+\int_{0}^{t} a(t, \omega) d t+\int_{0}^{t} B(t, \omega) d W_{t}$, where $a:[0,1] \times \Omega \rightarrow X$, $B:[0,1] \times \Omega \rightarrow L(X, X)$ are $(t, \omega)$-measurable, $\mathrm{F}_{t}$-adapted and $\int_{\Omega}^{t} \int_{0}^{t}\|a(t, \omega)\|_{M_{1}}{ }^{2} d P d t<\infty, B \in T G_{R}(L(X, X))$. Let also $f:[0,1] \times X \rightarrow X$, be such that $f:[0,1] \times M_{2} \rightarrow M_{2}, f_{t}^{\prime}, f_{T}^{\prime}$ and $f_{T T}^{\prime \prime}$ are continuous by the norm of $M_{1}\left(X \subset \overline{M_{2}} \subset M_{1}\right)$. Then, taking into consideration that the step functions for $T_{t} \in \bar{M}_{2}$ we can take $X$-valued, we have
The first five members of the aforementioned equality are functionals from the $X$-valued processes. Therefore, the stochastic integral $\int_{0}^{1} f_{T}^{\prime}\left(s, \xi_{s}\right) B(s) d W_{s}$ as the $X$-valued random process exists. Consequently, we have received the Ito formula for the Banach space-valued Ito process.

Theorem 2. Let $\xi_{t}=\xi_{0}+\int_{0}^{t} a(s, \omega) d s+\int_{0}^{t} B(s, \omega) d W_{s}$, where $a:[0,1] \times \Omega \rightarrow X \quad, \quad B:[0,1] \times \Omega \rightarrow L(X, X) \quad$ be $\quad(t, \omega)$ measurable, $\mathrm{F}_{t}$-adapted and $\int_{\Omega}^{t} \int_{0}^{t}\|a(t, \omega)\|_{M_{1}}{ }^{2} d P d t<\infty, B \in$ $T G_{R}(L(X, X))$. Let $f:[0,1] \times X \rightarrow X$ be such that $f:[0,1]$ $\times M_{2} \rightarrow M_{2}, f_{t}^{\prime}, f_{T}^{\prime}$ and $f_{T T}^{\prime \prime}$ are, continuous then

$$
f\left(t, \xi_{t}\right)=f\left(0, \xi_{0}\right)+\int_{0}^{t} f_{t}^{\prime}\left(s, \xi_{s}\right) d s+\int_{0}^{t} f_{T}^{\prime}\left(s, \xi_{s}\right) a(s) d s+\frac{1}{2} \int_{0}^{t} \sum_{m=1}^{\infty} f_{T T}^{\prime \prime}\left(s, \xi_{s}\right) B(s, \omega) x_{m} B(s, \omega) x_{m} d s,+\int_{0}^{t} f_{T}^{\prime}\left(s, \xi_{s}\right) B(s, \omega) d W_{s}
$$

We have chosen $\left(x_{n}\right)_{n \in N}$ such that $R x^{*}=\sum_{k=1}^{\infty}\left\langle x_{k}, x^{*}\right\rangle x_{k}, \quad \frac{1}{2} \int_{0}^{t} \sum_{m=1}^{\infty} f_{T T}^{\prime \prime}\left(s, \xi_{s}\right) B(s, \omega) x_{m} B(s, \omega) x_{m} d s$ is the same for all $x^{*} \in X^{*}$, and $\sum_{k=1}^{\infty}\left\|x_{k}\right\|^{2}<\infty$. Analogously of proposition 3 $\left(x_{n}\right)_{n \in N}$ such that $R x^{*}=\sum_{k=1}^{\infty}\left\langle x_{k}, x^{*}\right\rangle x_{k}$ from [3], we can prove, that the expression 


\section{Conclusion}

The theory of stochastic differential equations in Banach space is develops in three directions. The first direction is the case, when the stochastic integral of the equation is taken from Banach space valued non-anticipating random function by the real-valued Wiener process; the second direction is the case, when the stochastic integral of the equation is taken from operator-valued non-anticipating random function by the Wiener process in a Banach space; the third direction is the case, when the stochastic integral of the equation is taken from operator-valued (from Hilbert space to Banach space) non-anticipating random function by the canonical generalized Wiener process in Hilbert space. Analogous to the finite dimensional case, the Ito formula is one of the main tools in stochastic analysis in Banach space. In this paper we consider the second case and prove the Ito Formula in this case. Existence and uniqueness of solutions is considered in [12] for this case. As we mentioned above the first case is considered in [4] and the third case, when the Banach space has special geometry are considered in [1] and [2] . in [10] is considered the case, when the function $f$ maps from Banach space to real line.

\section{References}

[1] I. I. Belopolskaia, Yu. L. Daletzky (1978), Diffussion Process in Smooth Banach Spaces and Manifolds. (Russian) Trudy Moskov. Mat. Obshch. 37, p.107-141.

[2] Z.Brzez'niak, J.M.A.M. van Neerven, M.C. Veraar, L Weis (2008), Ito's Formula in UMD Banach Spaces and Regularity of Solutions of the Zakai Equation. Journal of Differential Equations v.245, 1, p.30-58.
[3] B. Mamporia (2000), On the Ito Formula in a Banach Space. Georgian Mathematical Journal vol.7, 1, p.155-168.

[4] B. Mamporia, Ito's formula in a Banach space. Bull. Georgian National Academy of Sciences, vol. 5, no. 3, 2011, 12-16.

[5] N. N. Vakhania, V. I. Tarieladze, and S. A. Chobanian (1985), Probability Distributions on Banach Spaces. Nauka, Moskow; The English translation: Reidel, Dordrecht, the Netherlands, 1987. p.482.

[6] B. Mamporia, On Wiener process in a Frechet space. Soobshch. Acad. Nauk Gruzin. SSR, 1977

[7] B. Mamporia, Wiener Processes and Stochastic Integrals in a Banach space. Probability and Ma thematical Statistics, Vol. 7, Fasc. 1 (1986), p.59-75.

[8] S. Kwapie'n and B. Szymanski, Some remarks on Gaussian measures on Banach space. Probab. Math. Statist. 1(1980), No. 1, p. 59-65

[9] B. Mamporia. Stochastic differential equation for generalized random processes in a Banach space. Theory of probability and its Applications, 56(4),602-620,2012, SIAM.Teoriya Veroyatnostei i ee Primeneniya, 56:4 (2011), 704-725.

[10] H.H. Kuo, Gaussian measures in Banach spaces. SpringerVerlag, Berlin, Heidelberg, New York, 1975.

[11] Da Prato G., Zabczyk J. Stochastic Differential Equations in Infinite Dimensions. Encyclopedia of Mathematics and its Aplications. Cambridge University Press, 1992.

[12] B. Mamporia. Stochastic differential equation driven by the Wiener process in a Banach space, existence and uniqueness of the generalized solution. Pure and Applied Mathematics Journal 2015:4(3):133-138. 